\title{
Beam steering application for W-band data links with moving targets in 5G wireless networks
}

\author{
Álvaro Morales ${ }^{1 *}$, Sebastián Rodríguez ${ }^{1}$, Omar Gallardo $^{1}$, \\ Juan Jose Vegas Olmos ${ }^{1}$, Idelfonso Tafur Monroy ${ }^{1,2}$ \\ 1. Technical University of Denmark, Department of Photonics Engineering, Oersteds Plads, Building 343, \\ Kongens Lyngby 2800, Denmark \\ 2. Saint-Petersburg National Research University of Information Technologies, Mechanics and Optics, \\ Kronverksky prospekt, Saint Petersburg 197101, Russian Federation \\ *Corresponding author, Email: alvi@fotonik.dtu.dk
}

\begin{abstract}
Ubiquitous broadband Internet access is one of the major goals of the next generation of wireless communications. However, there are still some locations where this is difficult to achieve. This is the case on moving vehicles and, particularly, on trains. Among the possible solutions to this problem, RoF (Radio-over-Fiber) architectures have been proposed as low-latency, cost-effective candidates. Two elements are introduced to extend the RoF approach. First, the carrier frequency is raised into the W-band $(75-110 \mathrm{GHz})$ to increase the available capacity. Second, a mechanical beam-steering solution based on a Stewart platform is adopted for the transmitter antenna to allow it to follow a moving receiver along a known path, thereby enhancing the coverage area. The performance of a system transmitting a $2.5 \mathrm{Gbit} / \mathrm{s}$ non-return-to-zero signal generated by photonic up-conversion over a wireless link is evaluated in terms of real-time BER (Bit Error Rate) measurements. The receiver is situated in different positions, and the orientation of the transmitter is changed accordingly. Values below the forward error correction limit for $7 \%$ overhead are obtained over a range of $60 \mathrm{~cm}$ around a center point situated $2 \mathrm{~m}$ away from the transmitter.
\end{abstract}

Keywords: 5G mobile communications, mechanical steering, millimeter-wave communications, photonic up-conversion, vehicular communications

Citation: Á. Morales, S. Rodríguez, O. Gallardo, et al. Beam steering application for W-band data links with moving targets in $5 \mathrm{G}$ wireless networks $[\mathrm{J}]$. Journal of communications and information networks, 2017, 2(2): 91-100.

\section{Introduction}

The huge growth in the existing number of personal mobile devices over the last few years has generated the need for high-speed, ubiquitous Internet access. One of the major goals of $5 \mathrm{G}$ (fifth Generation) mobile communications is to be able to connect to an end user at any location while assuring an accept-

Manuscript received Feb. 22, 2017; accepted Apr. 12, 2017

This work is supported by the Marie Skłodowska-Curie Innovative Training Network CELTA supported by the European Union's Horizon 2020 research and innovation programme (No. 675683), the Innovative Training Network FiWiN5G project founded by the European Union's Horizon 2020 research and innovation programme (No. 642355). 
able Quality of Service ${ }^{[1]}$. Nevertheless, some locations give rise to specific challenges due to their characteristics. In particular, high-speed trains with speeds of $200 \mathrm{~km} / \mathrm{h}$ or more are considered locations where the achievable quality of connection is not high enough to accommodate the traffic demands of the passengers ${ }^{[2,3]}$. There are some challenges that must be taken into account to maintain a connection with a bullet train ${ }^{[2,3]}$. First, wireless interfaces are needed to connect a moving vehicle with ground BSs (Base Stations). Moreover, the train may go through tunnels or through sparsely populated areas where the existing infrastructure is insufficient to provide continuous coverage. Another important issue is the handover between various BSs while the train is moving, which is a situation occurring more frequently for high-speed trains. Finally, both the metal structures of the carriages and the environmental vibrations result in additional attenuation of wireless signals.

Despite these constraints, efforts should still be made to provide high-speed access to passengers on trains, because there are large potential economic benefits from doing this ${ }^{[2]}$. However, it is insufficient to merely connect the passengers with BSs on the ground. In addition, both the high bandwidth and capacity demands of future applications are expected to be satisfied. As a consequence, millimeter wave (mm-wave) frequencies $(30-300 \mathrm{GHz})$ are gaining more attention for implementation in the last hop between the ground and a train ${ }^{[4]}$. This approach can be combined with the use of optical methods for signal generation, processing, and distribution. In this RoF (Radio-over-Fiber) scenario, more highly complex operations are carried out by a centralized station, which distributes the data to several RAUs (Radio Access Units) spread along the train tracks, serving as an interface between the network and the wireless transmission to the train. RoF has also been declared a promising option for implementing radio access networks in $5 \mathrm{G}$ communications ${ }^{[5,6]}$. Therefore, the integration of broadband railways communications into $5 \mathrm{G}$ networks can be achieved in practice by including them as clusters within optical net- works for mobile applications ${ }^{[7]}$.

Unfortunately, there are still some problems left to solve when operating at those frequencies. In particular, the high free-space path loss and the atmospheric absorption that occur and cause difficulties in implementing long-distance links ${ }^{[8]}$. Although attenuation is high at mm-wave frequencies, there are some frequency windows in which the atmospheric absorption is lower, offering high-capacity potential. One of these is the W-band $(75-110 \mathrm{GHz})$, which is considered one of the main candidates for radio frequency links ${ }^{[9-11]}$. The use of high gain directive antennas is a possible solution to attenuation. These antennas allow longer transmission distances to be achieved at the expense of focusing the radiated energy on reduced areas. Therefore, the ability to steer the beam direction is required to enhance the coverage region ${ }^{[12]}$. Several techniques have been proposed over the last few years, including mechanical steering, beamforming ${ }^{[13]}$, the use of reflect arrays ${ }^{[14]}$, and the use of lens antennas ${ }^{[15]}$.

In this article, a mechanical beam steering application, based on a Stewart platform, is proposed for use in the RAUs of an RoF architecture to provide high-speed Internet to moving targets in $5 \mathrm{G}$ wireless networks. In our RoF concept, radio carriers are not transported over the fiber, but rather, data is transmitted as a baseband signal and the radio carrier is generated on a photodiode that acts as a heterodyne mixer. Therefore, by employing a Stewart platform on which both the antenna and the photomixing element are placed, our solution combines the RoF method with beam steering that is independent of both the carrier frequency and the data transmission bandwidth. The remainder of the paper is organized as follows. In section 2, the main architectures for providing high-speed connections to trains that are reported in the literature are briefly described. Later, in section 3, our proposed architecture is presented by introducing both transmission at $\mathrm{W}$-band frequencies and antenna-steering capabilities to the RoF approach. Subsequently, an accurate mechanical beam steering application is outlined in section 4. In sections 5 and 6 , we present a real-time experi- 
ment and its results for the wireless transmission of a 2.5 Gbit/s NRZ (Non-Return-to-Zero) signal with a carrier frequency of $81 \mathrm{GHz}$ (W-Band) that validates the aforementioned architecture. The transmission antenna has the ability to steer the radiation beam to follow the receiver, which is situated in different positions.

\section{Architectures for railway communi- cations}

Several architectures have been proposed over the last few years for providing broadband internet to train passengers. The simplest solution consists of relying on the pre-existing cellular network on the ground outside the train ${ }^{[16]}$, which is known as a terminal-to-ground architecture. Passengers' mobile devices are directly connected to the BSs on ground. The most significant problem with this architecture is that trains pass through areas with coverage that is poor or even non-existent because of the geography. Furthermore, the signal is drastically attenuated by the train carriage, which has Faraday cage characteristics. Moreover, when a transition between cells occurs, the handover must be done for every mobile terminal on the train. As a consequence, train-toground architectures are preferred. In these architectures, the communications between end users and the access network are split into two segments: a wireless link between a BS on the ground and an AP (Access Point) on the train and an intra-train network connection connecting the passengers with the common $\mathrm{AP}^{[2,16]}$. Therefore, only the AP must be switched from one cell to another when a handover takes place, and better coverage is achieved since the train antenna is situated outside the metal structure of the train.

While WLAN IEEE 802.11 is the most widely used standard for intra-train networks, there are many possibilities for implementing the last hop between the train and the distributed networks:

- Satellite communications. These represent a traditional method of providing broadband access to moving vehicles. Satellite links have some important limitations, such as high latency and the need for a line of sight (reducing the coverage in cities and in tunnels). Although this technology is insufficient for achieving the broadband requirements of the future, it represents a good choice for use in sparsely populated areas where the deployment of terrestrial infrastructure is more difficult ${ }^{[3]}$. For instance, French TGV trains use satellites links to provide coverage in some areas.

- Railway cellular communications. Cellular communications with dedicated BSs distributed along the train track are another widespread solution. The GSM-R (GSM (Global System for Mobile Communication) for Railways) appeared as an extension of GSM and became the network standard for railway communications $^{[17]}$. Currently, it is unable to support increasing bandwidth demands, so it has been replaced by the $4 \mathrm{G}$ standard, LTE-R, which offers a better performance. The main advantage of LTE-R is the high capacity offered by $4 \mathrm{G}$ networks ${ }^{[17]}$. The AVE trains in Spain and the ICE 3 trains in Germany have embraced these solutions.

- Wireless LAN. It is possible to use the IEEE 802.11 standard to connect trains with ground BSs. Doing so provides both large capacities and reduced latencies ${ }^{[2]}$. The main disadvantages of this method are the small sizes of the cells, which result in greater numbers of handovers, and the high costs associated with the deployment of its associated infrastructure over the track. Some high-speed trains, such as the ETR 500 in Italy and the Acela in the US, have adopted this solution. As an alternative, WIMAX (Worldwide Interoperability for Microwave Access) has also been used as a standard for connecting high-speed trains, obtaining better performances than IEEE 802.11 solutions with larger cell sizes $^{[3]}$. For example, the Finnish high-speed train, Pendolino, relies on WIMAX for its communications.

- LCX (Leaky Coaxial Cable). This method is the preferred option for Japanese high-speed trains, such as the N700 Shinkansen. Radiating cables are laid along the tracks to provide communications with a mobile router aboard the $\operatorname{train}^{[18]}$. LCX provides a high capacity, although its capacity is lower than 
those of other solutions, including LTE-R and RoF.

- Radio-over-Fiber. Fast moving users architecture was proposed by Greve et al. ${ }^{[19]}$ as an RoF solution for providing connectivity to trains. The main concept of this method is the distribution of several RAUs along the tracks, which are fed by an optical ring network connected to a centralized control station. In the RAUs, the optical data is converted to electrical data and transmitted though the antennas. This is a cost-effective solution that provides high-capacity access. Its main limitation is its cell size. The THSR 700T train, used in both China and Taiwan, has adopted an RoF approach.

- CR (Cognitive Radio). PTC (Positive Train Control) is a system that is used to control and monitor trains in order to improve their safety. In addition to the data generated by the passengers, there is also train-related information, such as telematics, control, and signaling data, that needs to be transmitted. Several radio solutions have been considered to accomplish this, but CR has emerged as the leading candidate ${ }^{[3]}$. The outstanding characteristic of this system is its ability to constantly allocate free bandwidth slots using persistent parameter observation, including observation of the carrier frequency, bit rate, and modulation scheme parameters. This solution is used to enhance the train's security but not to provide Internet connections to the passengers.

Among all of the abovementioned architectures, RoF and LTE-R are the leading candidates for accommodating the high bandwidth demands of future networks, since they are the options that offer the highest capacities. The main advantage of RoF over LTE-R is that the deployment cost of the RAUs, which are not responsible of any data processing, is lower than the cost of the BSs in LTE, which are more complex. On the other hand, the cell size is much smaller in RoF than it is in LTE-R, resulting in a greater number of handovers. Even though the smaller cell radius appears to be a problem, it allows for more efficient use of the available frequency spectrum, since the same channel can be used by different RAUs that are separated by sufficient distances.

\section{$3 \quad$ Proposed architecture}

The architecture proposed in this work for the ground-to-train segment is shown in Fig. 1 and is composed of three elements:

- Control station. The control station serves as an interface between the aggregation network and the railways access network. It is responsible for both modulating the data and transmitting it via optical fibers to the RAUs. It utilizes its central view of the network to develop efficient algorithms for handovers between adjacent cells, since the order of the RAUs is known over the route of the $\operatorname{train}^{[4,20]}$. The signal generated at the control station is distributed to the RAUs through optical fibers.

- RAU (Radio Access Unit). The RAUs are spread along the train tracks. They receive data from the optical network and perform up-conversion from an optical baseband to a wireless carrier by seamlessly photomixing the data into any protocol or modulation format, thus both simplifying the architecture and reducing costs. Moreover, the wireless carrier selection is easily made based on the choice of the proper wavelength for the tunable local oscillator light source. After that, the signal is transmitted wirelessly to the train. Two key features are introduced: transmission at W-band frequencies $(75-110$ $\mathrm{GHz}$ ) and beam steering capabilities. Taking advantage of the fact that each RAU is dedicated exclusively to providing Internet to the train over a known path along the tracks, a beam steering application can be used to focus an RAU antenna's radiated energy while following the receiver mounted on the train. Combining this concept with transmission at mm-wave frequencies, both increases the capacity available and results in a promising method of overcoming the RoF scenario problems. Long distance links have previously been demonstrated at Wband frequencies ${ }^{[11]}$. Theoretically, cells with radii of around $100 \mathrm{~m}$ can be achieved, both increasing the cell size and reducing the number of handovers.

- Train access terminal. The train access terminal is the antenna on the train that recovers the wireless signal, which is subsequently demodulated. 
After that, this terminal serves as a bridge to the deployed WLAN IEEE 802.11 network, distributing repeaters to every carriage, as shown in Fig. 1(b). As a consequence, a passenger travelling on the train can connect their mobile device to the train's WLAN network, and the device will be forwarded to the global Internet through the RoF network described.
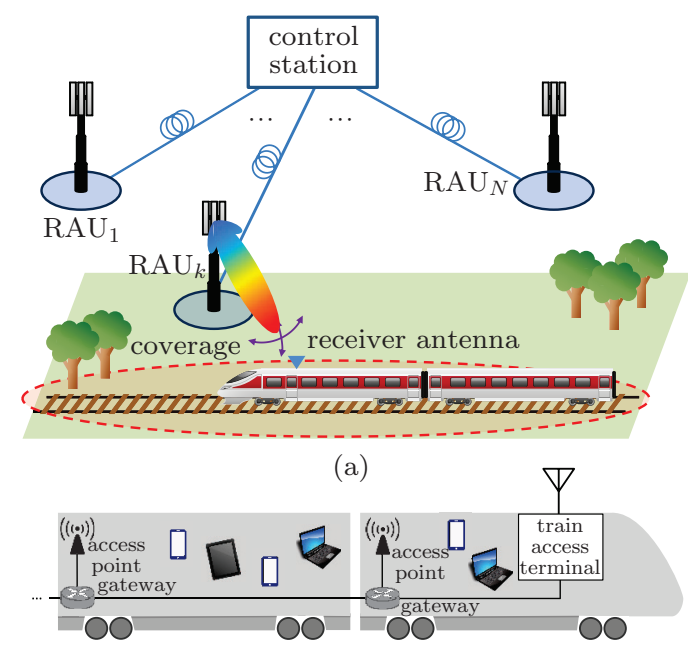

(b)

Figure 1 RoF architecture. (a) Centralized control station optically distributes data to RAUs spread along train tracks; (b) intra-train WLAN IEEE 802.11 network deployed inside train

There are two main issues that need to be addressed by this architecture: the handover process and train tracking. Dynamic network reconfiguration at mm-wave frequencies is a topic that has been widely discussed ${ }^{[21]}$. An RoF architecture for seamless communication using a carrier frequency of 60 $\mathrm{GHz}$ was proposed by Pleros et al. ${ }^{[4]}$. They verified the effectiveness of a handover algorithm operating between picocells for high mobility users. The antenna on the RAU has to be able to follow the AP mounted on the train. There is a lot of train-related information (e.g., position, speed, telematics) that also gets transmitted. This data can be used to define an effective algorithm for tracking the moving vehicle.

Although, for simplicity of exposition, the architecture has only been described under the consideration that data is transmitted from the control station to the passengers, communications are actually bidi- rectional. The antenna systems in the RAUs and on the train act as both transmitters and receivers. The remainder of this article will focus on testing and demonstrating the feasibility of the RAU scheme.

\section{Implemented beam steering system}

In the broadest sense, the term "beam steering" refers to the ability to adjust the radiation beam pointing out of an antenna. Several techniques have been used to accomplish this over the years ${ }^{[12]}$. Beamforming uses an array of antenna elements with their radiated fields combined in such a way that the desired radiation pattern is generated in the far field. In analog beamforming, this result is achieved by adjusting the gains and phases of the signals feeding the array, whereas in digital beamforming, it is achieved through digital processing ${ }^{[13,22]}$.

Although antenna arrays have been widely used for beam steering in other spectrum regions, the characteristics of the mm-wave range make using them complex and costly. Reflectarrays represent an alternative to these. They consist of an array of reflecting elements in which the phase shift provided by each element can be controlled. As a consequence, the reflected wave can have the desired properties ${ }^{[14]}$. This is a more feasible solution for high frequencies, but both the achieved steering speed and accuracy are lower. Another option is the use of an array of independent antennas positioned at the focal plane of a lens, illuminating it from different positions. Its beam direction can be modified by switching the radiating element among all the antennas in the array ${ }^{[15]}$. The main drawback of this solution is its inefficiency, since only one element is radiating at any time.

A trivial solution, which can overcome the complexity constraints of beam steering techniques at mm-wave frequencies, is mechanical movement of the radiating elements. Mechanical steering refers to turning an antenna to face the direction of interest. This method still carries some limiting factors, such as antenna size and steering speed. However, mechanical steering is suitable for aiming at 
Table 1 Comparison of beam steering techniques at mm-wave frequencies

\begin{tabular}{ccccccc}
\hline technique & inserttion loss & complexity & cost & resolution & speed & frequency dependence \\
\hline analogue beamforming & high & moderate & high & high & fast & high \\
digital beamforming & high & high & high & very high & fast & limited by adc \\
refelctarray & medium & moderate & high & high & fast & high \\
lens antenna & low & low & medium & high & fast & high \\
mechanical steering & low & low & low & continuous & slow & none \\
\hline
\end{tabular}

a moving target with a known path while transmitting at mm-wave frequencies, since the antennas can be constructed small enough to accomplish this. The main advantages of mechanical techniques are reduced cost and the fact that the radiation characteristics are maintained over the whole steering range. Despite their simplicity, there have not been many feasibility studies performed on using mechanical steering methods at mm-wave frequencies. Tab. 1 shows a comparison of the aforementioned methods.

The solution proposed for beam steering at $\mathrm{W}$ band frequencies consists of placing the transmitter antenna on a Stewart platform. This device is a robot based on a 6 -axis (i.e., $x, y, z$, pitch, roll, and yaw) actuator system arranged in parallel to two platforms and utilizing direct drive brushless DC motors (Physik Instrumente PI H-811.D2). The main advantages of this robot are its high resolution (40 $\mathrm{nm}$ for a single actuator) and its repeatability $(0.06 \mu \mathrm{m})$. A horn antenna operating in the W-band is mounted on a Stewart platform, as shown in Fig. 2, which includes the coordinate system used. This antenna can be moved with six degrees of freedom: three translational axes $(x, y$, and $z)$ and three rotational axes ( $w$, or azimuth angle; $v$, or elevation angle; and $u$ angle).

\section{Experimental setup description}

The experimental setup is shown in Fig. 3. At the transmitter side, a PPG (Pulse Pattern Generator) provides a $2.5 \mathrm{Gbit} / \mathrm{s}\left(2^{15}-1\right)$-bit long pseudorandom bit sequence (PRBS15) NRZ signal to a SFP+ module. The optical output the $\mathrm{SFP}+$ module, which has an extinction ratio of $9 \mathrm{~dB}$, is launched into $10 \mathrm{~km}$ of standard single-mode fiber, represent-

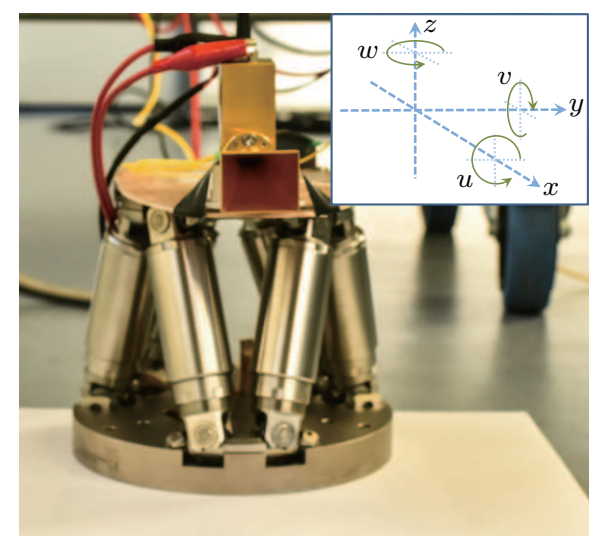

Figure 2 Stewart platform coordinate system: platform can be moved with six degrees of freedom inside the train, including the standard $x y z$ coordinate system directions and the rotation coordinates $u, v$, and $w$.

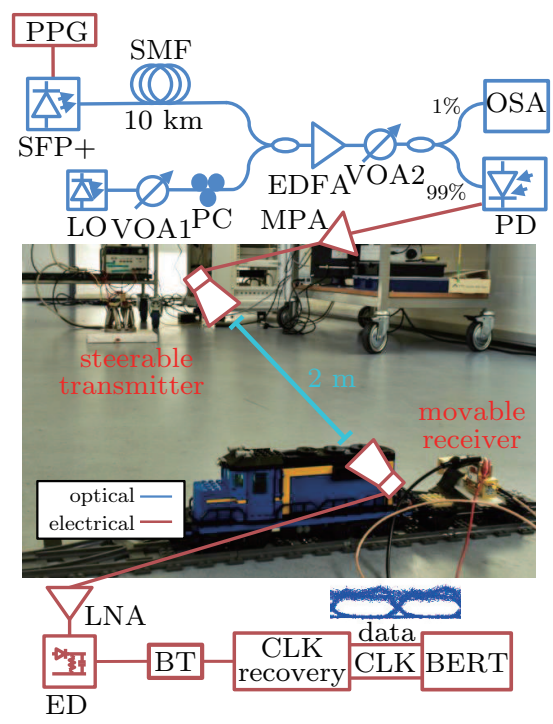

Figure 3 Experimental setup. PPG: pulse pattern generator, $\mathrm{SFP}+$ : enhanced small form factor pluggable, SMF: single mode fiber, LO: local oscillator, VOA: variable optical attenuator, PC: polarization controller, EDFA: erbium-doped fiber amplifier, OSA: optical spectrum analyzer, PD: photodiode, MPA: medium power amplifier, LNA: low noise amplifier, ED: envelope detector, BT: bias tee, CLK: clock, BERT: bit-errorrate tester. 
ing an optical link between the control station and the RAU. At that point, the signal is coupled with an external cavity laser that is acting as a tunable LO (Local Oscillator). The frequency of the LO is modified to create a frequency difference of $81 \mathrm{GHz}$ with the data signal. The power is controlled by a VOA (Variable Optical Attenuator), so the optical power of both branches is approximately the same before coupling. Next, an EDFA is implemented to raise the optical power of both the data signal and the LO simultaneously. Immediately after that, a second VOA is used to control the optical power incident on the photodiode $P_{\mathrm{PD}}$ to perform real time measurements of the BER. In the $\mathrm{PD}$, the optical up-conversion process takes place, and, as a result, an RF signal is generated with a (W-band) carrier frequency of $81 \mathrm{GHz}^{[6,10]}$. In the electrical domain, the signal is amplified to $8 \mathrm{~dB}$ by a medium power amplifier and transmitted using a horn antenna with a gain of $24 \mathrm{dBi}$.

Afterwards, the recovered signal is amplified by an LNA with a gain of $40 \mathrm{~dB}$ and then demodulated with an ED (Envelope Detector) based on a Schottky diode with a nominal bandwidth of $3 \mathrm{GHz}$. Finally, a clock recovery stage provides both the data and timing signals to a BERT (Bit Error Rate Tester) to perform real-time measurements.

In the experiment described above, the environmental aspects are limited to the case of a moving target. There are still aspects of vibrations, wireless channels variations, and other phenomena that are left for matters of further study. In a more realistic deployment, the performance of our proposed beam steering method depends on the relationships among the speed of the moving object, the employed platform's adjustment speed, and the delays caused by any feed-back mechanism. However, the scalability of our system can be verified. Stewart platforms that are currently available on the market can achieve angular velocities of up to $34^{\circ} \%$ s. In our experiment, this implies that the maximum velocity of a moving receiver, disregarding the feedback delay, is $4.3 \mathrm{~km} / \mathrm{h}$. W-band wireless links have been demonstrated for distances of up to $220 \mathrm{~m}^{[11]}$, which means that an antenna unit can be situated $200 \mathrm{~m}$ away from the tracks and cover a $60 \mathrm{~m}$ diameter cell. In this scenario, a Stewart platform could follow a bullet train travelling with a speed of up to $340 \mathrm{~km} / \mathrm{h}$.

\section{$6 \quad$ Experimental results}

To demonstrate the concepts addressed in this paper, real-time BER measurements are taken in 13 different receiver positions along the functional range, as shown in Fig. 4(c), between $\pm 30 \mathrm{~cm}$. For each position, the $\lg (B E R)$ is plotted with respect to the optical power incident on the photodiode $P_{\mathrm{PD}}$. The surface containing the results, as shown in Fig. 5, is compared with the BER limit of $3.8 \times 10^{-3}$ using a typical FEC (Forward Error Correction) scheme with $7 \%$ overhead.

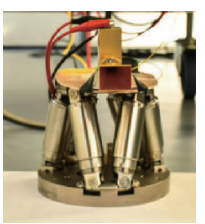

(a)

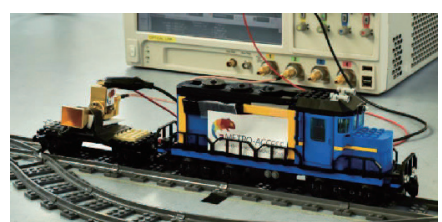

(b)

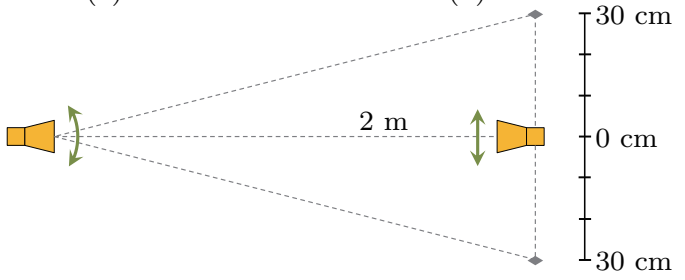

(c)
Figure 4 Experimental setup. (a) The transmitter antenna is mounted on a Stewart platform that can be steered with six degrees of freedom; (b) the receiver antenna is placed on a platform which allows vertical displacement with respect the transmitter; (c) overall scenario

In consideration of these results, there are several conclusions that can be drawn. When the receiver is at $0 \mathrm{~cm}$, a small BER (close to error-free transmission) is achieved with $P_{\mathrm{PD}}=2 \mathrm{dBm}$. Even when reducing the optical power to less than $0 \mathrm{dBm}$, the BER remains below the FEC limit. When the distance increases, a higher power is needed to achieve the same BER values. There are two main reasons for this behavior. First, the distance between the antennas increases, so, according to the Friis transmis- 
sion equation, the power received decreases due to attenuation. Second, directive antennas are used in both transmission and reception, which means that a lower gain is achieved because of misalignment, since the receiver antenna is not pointing directly at the transmitter.

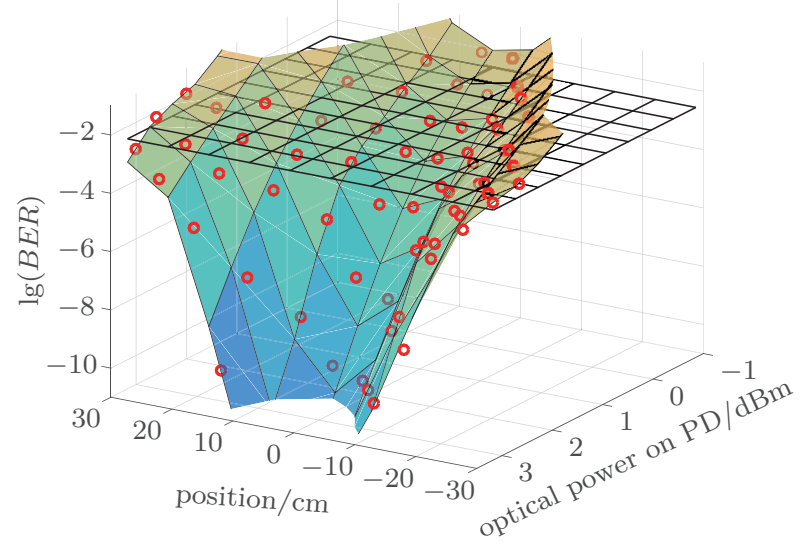

Figure 5 Experimental results of BER with respect optical power incident on photodiode with receiver situated at different positions

An important parameter to measure is the sensibility of the system, defined as the minimum optical power needed for the photonic up-conversion process to guarantee that the possible errors made will allow for recovery. Fig. 6 shows the sensibility, calculated graphically as the curve where the BER surface intersects with the limit determined by the FEC techniques. For a receiver situated close to the center, values below $0 \mathrm{dBm}$ are achieved with a minimum of around $-0.5 \mathrm{dBm}$. Although the sensibility increases for larger displacements, successful communication can still be achieved with an optical power of $2.75 \mathrm{dBm}$.

Finally, Fig. 7 shows the BER with respect to the receiver position for different values of optical power. In other words, it depicts the range in which the receiver can be moved depending on the available optical power. With $P_{\mathrm{PD}}=0 \mathrm{dBm}$, the receiver can be moved when it is between $-5 \mathrm{~cm}$ and $+10 \mathrm{~cm}$. When the power increases by $1 \mathrm{~dB}$, the functional range becomes $\pm 15 \mathrm{~cm}, \pm 20 \mathrm{~cm}$ in the case of $P_{\mathrm{PD}}=2 \mathrm{dBm}$, and the entire range is covered when $P_{\mathrm{PD}}=3 \mathrm{dBm}$.

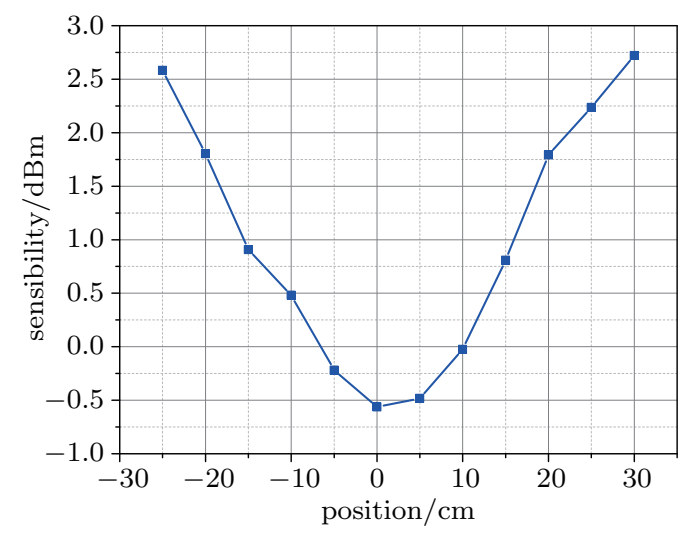

Figure 6 System sensibility, defined as the minimum optical power needed before the photonic up-conversion for successful communication

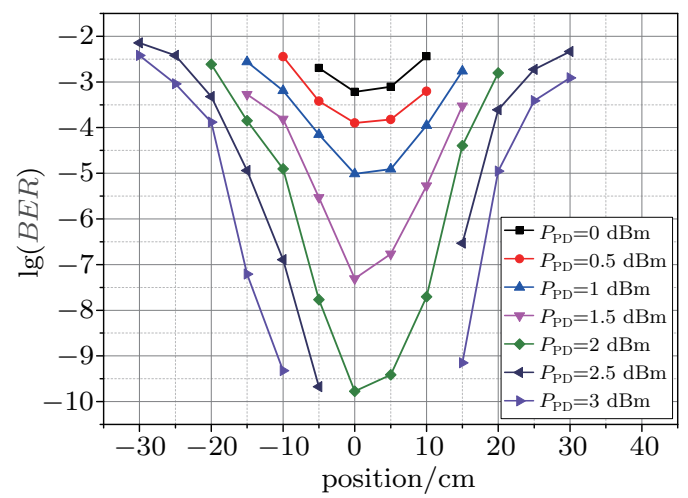

Figure 7 Experimental results of BER with respect the receiver position for a constant optical power incident on the photodiode

These results confirm that our proposed beam steering solution can provide foundations for applications in which the W-band band is used for high capacity wireless data transmission, such as last mile broadband access and high-speed wireless connections to trains, vehicles, or other moving objects. Moreover, it can provide foundations for applications in both industrial wireless communications control systems and robotic communication.

\section{Conclusions}

In this paper, we presented an architecture for delivering W-band mobile communications to the moving receivers of trains. The key features incorporated in this work are wireless transmissions in the $\mathrm{W}$-band to increase the capacity and beam steering capability for the transmitter antenna to follow 
the receiver over a known path, increase the coverage area, and optimize the power budget. Real-time measurements for the transmission of a $2.5 \mathrm{Gbit} / \mathrm{s}$ NRZ signal at a carrier frequency of $81 \mathrm{GHz}$ were taken at different positions of the receiver, with the transmitter, mounted on a Stewart platform, having the ability to track the receiver. With an optical power of $3 \mathrm{dBm}$ before the photonic up-conversion, BER values below the FEC limit were achieved over a range of $60 \mathrm{~cm}$ of vertical displacement for the receiver antenna around a center position situated $2 \mathrm{~m}$ away from the transmitter. Our results serve as engineering guidelines for the design of future communications systems that provide broadband connections to trains using an RoF approach at millimeter-waves frequencies.

\section{References}

[1] C. X. Wang, F. Haider, X. Fao, et al. Cellular architecture and key technologies for $5 \mathrm{G}$ wireless communication networks [J]. IEEE communications magazine, 2014, 52(2): 122-130.

[2] D. T. Fokum, V. S. Frost. A survey on methods for broadband Internet access on trains [J]. IEEE communications surveys \& tutorials, 2010, 12(2): 171-185.

[3] S. Banerjee, M. Hempel, H. Sharif. A survey of wireless communication technologies \& their performance for high speed railways $[\mathrm{J}]$. Journal of transportation technologies, 2016, 6(1): 15-29.

[4] N. Pleros, K Vyrsokinos, K. Tsagkaris, et al. A $60 \mathrm{GHz}$ radio-over-fiber network architecture for seamless communication with high mobility [J]. Journal of lightwave technology, 2009, 27(12): 1957-1967.

[5] J. J. Vegas Olmos, I. Tafur Monroy. Reconfigurable radio-over-fiber networks [J]. IEEE/OSA journal of optical communications and networking, 2015, 7(11): B23B28

[6] S. Rodriguez, S. Rommel, J. J. Vegas Olmos, et al. Reconfigurable radio access unit to dynamically distribute W-band signals in $5 \mathrm{G}$ wireless access networks $[\mathrm{J}]$. Optical switching and networking, 2017, 24: 21-24.

[7] J. J. Vegas Olmos, G. Rodes, I. Tafur Monroy. Optical switching for dynamic distribution of wireless-over-fiber signals in active optical networks [J]. Journal of optical communications and networking, 2012, 4(8): 622-627.

[8] T. S. Rappaport, S. Sun, R. Mayzus, et al. Millimeter wave mobile communications for $5 \mathrm{G}$ cellular: it will work! [J]. IEEE access, 2013, 1: 335-349.

[9] I. S. Amiri, S. E. Alavi, S. M. Idrus, et al. W-band
OFDM transmission for radio-over-fiber link using solitonic millimeter wave generated by MRR [J]. IEEE journal of quantum electronics, 2014, 50(8): 622-628.

[10] L. Cavalcante, S. Rommel, S. Rodriguez, et al. On the capacity of radio-over-fiber links at the W-band $[\mathrm{J}]$. Optical and quantum electronics, 2016, 48(5): 1-10.

[11] S. Rommel, S. Rodriguez, L. Chorchos, et al. Outdoor W-band hybrid photonic wireless link based on an optical SFP+ module [J]. IEEE photonics technology letters, 2016, 28(21): 2303-2306.

[12] I. Uchendu, J. R. Kelly. Survey of beam steering techniques available for millimeter wave applications $[\mathrm{J}]$. Progress in electromagnetics research B, 2016, 68(1): 3554.

[13] R. Bonjour, M. Singleton, S. A. Gebrewold, et al. Ultrafast millimeter wave beam steering [J]. IEEE journal of quantum electronics, 2016, 52(1): 1-8.

[14] A. Tamminen, J. Ala-Laurinaho, D. Gomes-Martins, et al. Reflectarray for $120-\mathrm{GHz}$ beam steering application: design, simulations, and measurements $[\mathrm{C}] / /$ Proceedings SPIE 8362, Passive and Active MillimeterWave Imaging XV, Baltimore, USA, 2012: 836205.

[15] J. Ala-Laurinaho, A. Karttunen, J. Säily, et al. Mmwave lens antenna with an integrated LTCC feed array for beam steering $[\mathrm{C}] / /$ Fourth European Conference on Antennas and Propagation (EuCAP), Barcelona, Spain, 2010: 1-5.

[16] Y. Q. Zhou, Z. G. Pan, J. L. Hu, et al. Broadband wireless communications on high speed trains $[\mathrm{C}] / / 20$ th Annual Wireless and Optical Communications Conference (WOCC), Newark, USA, 2011: 1-6.

[17] A. Sniady, J. Soler. Performance of LTE in high speed railway scenarios [C]//International Workshop on Communication Technologies for Vehicles, Villeneuve d' Ascq, France, 2013: 211-222.

[18] M. Terada, F. Teraoka. Providing a high-speed train with a broadband and fault tolerant IPv4/6 NEMO environment $[\mathrm{C}] / /$ Globecom Workshops (GC Wkshps), Anaheim, USA, 2012: 1052-1056.

[19] F. de Greve, B. Lannoo, L. Peters, et al. Famous: a network architecture for delivering multimedia services to fast moving users $[\mathrm{J}]$. Wireless personal communications, 2005, 33(3-4): 281-304.

[20] B. Lannoo, D. Colle, M. Pickavet, et al. Radio-overfiber-based solution to provide broadband Internet access to train passengers [J]. IEEE communications magazine, 2007, 45(2): 56-62.

[21] J. J. Vegal Olmos, T. Kuri, K. Kitayama. Dynamic reconfigurable WDM 60-GHz millimeter-waveband radioover-fiber access network: architectural considerations and experiment $[\mathrm{J}]$. Journal of lightwave technology, 2007, 25(11): 3374-3380.

[22] T. Do-Hong, P. Russer. Signal processing for wideband smart antenna array applications [J]. IEEE microwave magazine, 2004, 5(1): 5767 . 


\section{About the authors}

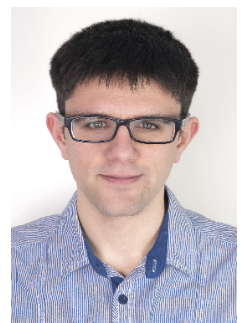

Álvaro Morales [corresponding author] was born in León, Spain. He received his B.Sc. in telecommunications technologies engineering from the University of Valladolid (UVa) in 2014, and his M.Sc. in telecommunications engineering from UVa in 2016. He started his PhD studies at the Technical University of Denmark (DTU) in 2016. In his research, he works on beam steering at mm-waves and sub-terahertz frequencies. (Email: alvi@fotonik.dtu.dk)

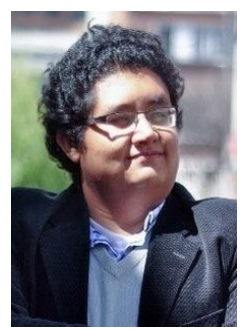

Sebastián Rodríguez was born in Bogotá, Colombia. He received his B.Sc. and M.Sc. degrees in electronic engineering from the Pontificia Universidad Javeriana in 2011 and 2014, respectively. Currently, he is working as a Marie Curie Early Stage Researcher (ESR) at the Technical University of Denmark (DTU), under the FiWiN5G project. In his research, he works on the switching of W-band signals for active and reconfigurable delivery. (Email: juse@fotonik.dtu.dk)

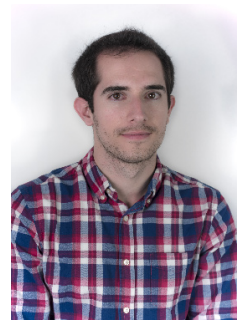

Omar Gallardo was born in Palencia, Spain. He received his B.Sc. in electronics and automation engineering from the University of Valladolid (UVa) in 2015. Currently studying a M.Sc. degree in electrical engineering with the study line in automation and robot technology at the Technical University of Denmark. (Email: omagal@student.dtu.dk)

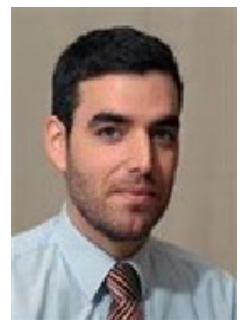

Juan Jose Vegas Olmos received the Ph.D. degree from TU Eindhoven, Eindhoven, the Netherlands, in 2006. He holds a BSc and MSc in electronic engineering, a BA in economics, and an MBA. In 2011 he joined the Technical University of Denmark, where since 2013 he is an associate professor at the Department of Photonics Engineering. (Email: jjvo@fotonik.dtu.dk)

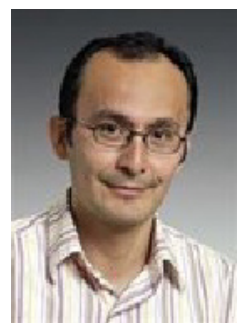

Idelfonso Tafur Monroy received his M.Sc. degree in multi-channel telecommunications from the Bonch-Bruevitch Institute of Communications, St. Petersburg, Russia, in 1992. In 1999, he received the Ph.D. degree from the Electrical Engineering Department, Eindhoven University of Technology, the Netherlands. He is currently a professor at the Department of Pho- tonics Engineering, Technical University of Denmark. (Email: idtm@fotonik.dtu.dk) 